very dense (and expensive) screening tools. To the extent that rare alleles are important to schizophrenia, study designs based on a naive 'common disease-common allele' model will yield variable and non-replicable results (King et al, 2006).

Characteristic patterns of age at onset, gender differences and brain changes associated with schizophrenia are fully compatible with causal influences of rare severe-effect events, either genetic or epigenetic. Each such event alters the expression, timing or function of one of a very large number of genes. The products of these genes converge in common pathways. Aberrations of a pathway by any of multiple mechanisms may lead to clinically similar disorders.

Crow's proposition that schizophrenia arises from the disruption of uniquely human genetic elements is very appealing. This premise, however, need not narrow the search for causes, genetic, epigenetic or environmental. Human speciation likely occurred primarily as a result of regulatory changes in genes, rather than common polymorphisms leading to changes in gene sequence (King \& Wilson, 1975). The extraordinary number of repeated elements in the human genome gave rise to a vast number of new genes and regulatory mechanisms. Their architecture also created an increased risk for copying errors. Thus, one cost of the genomic complexity that enabled human brain development may be a de novo error rate that results in the maintenance of schizophrenia in the population.

Autism has recently been shown to be associated with a significantly increased frequency of rare de novo mutations (Sebat et al, 2007). These results presage the identification of many more rare mutations associated with other neurodevelopmental illnesses, as advances in technology enable the detection of ever-smaller genomic lesions. The ultimate resolution of this debate lies in gene discovery, for which we encourage the application of study designs most likely to be fruitful.

Craddock, N., O'Donovan, M. C. \& Owen, M. J. (2007) Phenotypic and genetic complexity of psychosis. Invited commentary on . . . Schizophrenia: a common disease caused by multiple rare alleles. British Journal of Psychiatry, $190,200-203$.

King, M. C. \& Wilson, A. C. (1975) Evolution at two levels in humans and chimpanzees. Science, 188, 107-116.

King, M. C., Ahsan, H. \& Susser, E. (2006) Designs for the genomic era. In Psychiatric Epidemiology: Searching for the Causes of Mental Disorders (eds E.

Susser, S. Schwartz, A. Morebia, et al), pp. 40I-4I2.

Oxford University Press.
Sebat, J., Lakshmi, B., Malhotra, D., et al (2007) Strong association of de novo copy number mutations with autism. Science, 316, 445-449.

Jon McClellan Department of Psychiatry,

University of Washington, Seattle,WA 98195, USA.

Email: drjack@u.washington.edu

Ezra Susser Department of Epidemiology, Mailman School of Public Health, Department of Psychiatry, Columbia University, and New York State Psychiatric Institute, USA

Mary-Claire King Department of Genome Sciences and Department of Medicine (Medical Genetics), University of Washington, USA

doi: 10.I192/bjp.191.2.180a

\section{Anticipation and the genetics of psychosis}

A theoretical model should explain all of the observed facts. Both the model of schizophrenia which proposes many genes of small effect (McClellan et al, 2007) and that which proposes few gene mutations of large effect (Craddock et al, 2007) explain many of the observed facts. In particular, the model of many genes of small effect explains the observed spectrum of mental illness, from bipolar disorder, through schizoaffective disorder to schizophrenia.

We have both observed schizophrenia occurring in particular families. One of us (M.A.) has studied a number of SouthAsian families with multiple members with schizophrenia. In some of these families, patients of a second generation developed the illness at a much younger age than their parents and their illness was more severe. Thus far we have assumed that these observations were related to the concentration of many genes of small effect within these families.

One of us (M.B.-P.) has also observed the same effect, known as anticipation, in a group of families in Slovenia. Thirty-six parent-offspring pairs with schizophrenia were studied. First hospital admission was used as a proxy for disease onset. In the offspring group, mean age at onset was identified as 23.5 years whereas this was 39.6 years in the parent group. There was a higher mean total number of days of hospitalisation in the first 5 years of treatment in the offspring group (223 v. 161), and a higher mean number of hospitalisations over the same period in the offspring (7.27 v. 7.51) (both results statistically significant). These two measures were used as a proxy for increased intensity of illness. The offspring had a higher level of education but demonstrated fewer working years and had fewer children (Blinc, 2002).
What arises is the question of whether the 'many genes of small effect' or the 'few genes of large effect' model is best suited to explaining this observation of anticipation of schizophrenia.

Blinc, M. (2002) Druzinsko pojavljanji schizophrenijeZnacilinosti starsev in njihovih potomcev, obolelih za schizofrenijo. The Appearance of Schizophrenia in Families. [The Characteristics of the Illness in the Parents and their Offspring] (in Slovenian). Masters Thesis, University of Ljubljana.

Craddock, N., O'Donovan, M. C. \& Owen, M. J. (2007) Phenotypic and genetic complexity of psychosis. Invited commentary on . . . Schizophrenia: a common disease caused by multiple rare alleles. British Journal of Psychiatry, 190, 200-203.

McClellan, J. M., Susser, E. \& King, M. C. (2007) Schizophrenia: a common disease caused by multiple rare alleles. British Journal of Psychiatry, 190, 194-199.

M. Blinc-Pesek University of Lubljana, Slovenja

M. Agius Bedfordshire Centre for Mental Health Research in Association with the University of Cambridge, c/o Weller Wing, Bedford Hospital, UK. Email: Mark.Agius@blpt.nhs.uk

doi: 10.II92/bjp.19I.2.18I

\section{Attention-deficit hyperactivity disorder and mood disorders in adults}

Asherson et al (2007) raise some important issues regarding adult attention-deficit hyperactivity disorder (ADHD). They state that some symptoms of bipolar disorder are similar to those of ADHD but the distinction is not difficult. However, although ADHD and classic euphoric mania (bipolar I) may be distinct, differentiation of ADHD and bipolar disorder may be difficult, especially in bipolar II, bipolar-spectrum disorder and episodes of mixed symptomatology. At times, it may be almost impossible to discriminate solely by symptoms. Irritability, excessive activity, impulsive behaviour, poor judgement and denial of problems are characteristic of both ADHD and bipolar disorder, thus making diagnosis difficult. The two also clearly occur together in some individuals: the reported overall lifetime prevalence of comorbid ADHD in people with bipolar disorder is $9.5 \%$ (Nierenberg et al, 2005); comorbidity with unipolar disorder is also frequent.

Asherson et al state that ADHD is a persistent trait whereas bipolar disorder is episodic. However, inter-episodic symptoms are common in bipolar disorder and the course of both bi- and unipolar disorder is frequently chronic; for example, up to $13 \%$ of people with bipolar disorder report continuous cycling without a well phase 
and $54 \%$ are not fully euthymic between episodes (Kupka et al, 2001).

Children of mothers with bipolar I disorder have increased rates of both unipolar disorder and ADHD, further suggesting a neurobiological overlap of these three diagnoses. Hirshfeld-Becker et al (2006) report significantly higher rates $(23.5 \%)$ of ADHD in offspring of parents with bipolar disorder compared with psychiatric comparison parents $(8.4 \%)$ and non-psychiatric comparison parents $(4.2 \%)$.

Drug treatments also overlap. Stimulanttype medication has been used in bipolar depression, and newer medications such as atomoxetine have similar pharmacological characteristics to some antidepressants (Lydon \& El-Mallakh, 2006). Catecholaminergic antidepressants are not only potentially of benefit in ADHD but may be less likely to destabilise bipolar disorder.

There is thus a clinical and neurobiological overlap between ADHD, bipolar and unipolar disorder. Asherson et al's timely editorial has reminded us that ADHD in adults should not be overlooked and that further research is needed to clarify its impact on other adult psychopathology and comorbidity, particularly in mood disorders.

Asherson, P., Chen, W., Craddock, B., et al (2007) Adult attention-deficit hyperactivity disorder: recognition and treatment in general adult psychiatry British Journal of Psychiatry, 190, 4-5.

Hirshfeld-Becker, D. R., Biederman, J., Henin, A., et al (2006) Psychopathology in the young offspring of parents with bipolar disorder: a controlled pilot study. Psychiatry Research, 145, 155-167.

Kupka, R.W., Bolen, W. A., Altshuler, L. L., et a (200I) The Stanley Foundation Bipolar Network. 2. Preliminary summary of demographics, course of illness and response to novel treatments. British Journal of Psychiatry, 178 (suppl. 4I), sl77-sl83.

Lydon, E. \& El-Mallakh, R. S. (2006) Naturalistic long term use of methylphenidate in bipolar disorder. Journal of Clinical Psychopharmacology, 26, 516-518.

Nierenberg, A. A., Miyahara, S., Spencer, T., et a (2005) Clinical and diagnostic implications of lifetime attention-deficit/hyperactivity disorder comorbidity in adults with bipolar disorder: data from the first 1000

STEP-BD participants. Biological Psychiatry, 57, 1467-1473.

A. J. Kuan Department of Psychiatry, University of British Columbia, 2255 Wesbrook Mall, Vancouver BC, V6T 2AI, Canada. Email: anniekuan@gmail.com

A. H. Young Department of Psychiatry,

University of British Columbia, Canada

doi: 10.1192/bjp.19I.2.18la
Authors' reply: Kuan \& Young point out that further research into the role of mood symptoms in attention-deficit hyperactivity disorder (ADHD) is essential. In a recent study of 141 adults with ADHD, $95 \%$ were found to have mood symptoms, chiefly mood instability (Kooij, 2007). We observe that in adult ADHD mood instability frequently responds to stimulants over the same time course as core ADHD symptoms, an observation reported by others. This has led to the suggestion that mood dysregulation might represent a core impairment in adult ADHD, perhaps related to the same processes that cause dysregulation of other executive processes.

Despite these observations the relationship of ADHD to mood disorders is controversial. The controversy has arisen in the context of paediatric bipolar disorder, where the distinction from ADHD is made difficult if one chooses to view irritability as a sufficient manifestation of bipolar disorder and if the requirement for episodicity is not strictly applied. However, available validation studies for the construct of paediatric bipolar disorder use elation and/or grandiosity as cardinal symptoms, rather than irritability. Narrowly defined paediatric bipolar disorder can be differentiated from ADHD, shows longitudinal stability and has plausible familial aggregation patterns (Geller \& Tillman, 2005; Geller et al, 2006). Recent evidence suggests that the narrowly defined disorder can be distinguished at the behavioural and electrophysiological level from broadly construed disorder (Rich et al, 2007). Conversely, it has been argued that the intensity of irritability (Mick et al, 2005) and its temporal pattern (chronic or episodic) can distinguish paediatric bipolar disorder from ADHD (Leibenluft et al, 2006). The family study of Hirschfeld-Becker et al (2006) is intriguing, yet the sample size is small (12 families with bipolar I disorder, 11 with bipolar II disorder), and further work is needed to clarify the rates of ADHD among relatives with narrowly defined $v$. broadly defined bipolar disorder.

One of the main questions to be addressed relates to how valid a diagnostic concept broadly defined bipolar disorder is, or whether mood instability/irritability in the presence of ADHD may be more adequately described by a new dimension, such as mood dysregulation (Brotman et al, 2006). Until the relevant empirical data become available, we see merit in maintaining the classic definition of mania, so that a diagnosis of bipolar disorder requires euphoria, grandiosity and episodicity, and the differential between ADHD and bipolar disorder remains explicit.

Brotman, M. A., Schmajuk, M., Rich, B. A., et al (2006) Prevalence, clinical correlates, and longitudinal course of severe mood dysregulation in children. Biological Psychiatry, 60, 991-997.

Geller, B. \& Tillman, R. (2005) Prepubertal and early adolescent bipolar I disorder: review of diagnostic validation by Robins and Guze criteria. Journal of Clinical Psychiatry, 66 (suppl. 7), 2I-28.

Geller, B., Tillman, R., Bolhofner, K., et al (2006) Controlled, blindly rated, direct-interview family study of a prepubertal and early-adolescent bipolar I disorder phenotype: morbid risk, age at onset, and comorbidity. Archives of General Psychiatry, 63, I130-1138.

Hirshfeld-Becker, D. R., Biederman, J., Henin, A. et al (2006) Psychopathology in the young offspring of parents with bipolar disorder: a controlled pilot study. Psychiatry Research, 145, 155-167.

Kooij, J. J. S. (2007) ADHD in Adults: Clinical Studies on Assessment and Treatment. PhDThesis, Radboud University, Nijmegen.

Leibenluft, E., Cohen, P., Gorrindo, T., et al (2006)

Chronic versus episodic irritability in youth: community-based, longitudinal study of clinical and diagnostic associations. Journal of Child and Adolescent Psychopharmacology, 16, 456-466.

Mick, E., Spencer, T., Wozniak, J., et al (2005) Heterogeneity of irritability in attention-deficit/ hyperactivity disorder subjects with and without mood disorders. Biological Psychiatry, 58, 576-582.

Reimherr, F. W., Williams, E. D., Strong, R. E., et al (2007) A double-blind, placebo-controlled, crossover study of osmotic release oral system methylphenidate in adults with ADHD with assessment of oppositional and emotional dimensions of the disorder. Journal of Clinical Psychiatry, 68, 93-10।.

Rich, B. A., Schmajuk, M., Perez-Edgar, K. E., et a (2007) Different psychophysiological and behaviora responses elicited by frustration in pediatric bipolar disorder and severe mood dysregulation. American Journal of Psychiatry, 1634, 309-317.

P. Asherson MRC Social, Genetic and Developmental Psychiatry Centre, Institute of Psychiatry, Denmark Hill, London SE5 8AF, UK Email: P.Asherson@iop.kcl.ac.uk

A. Stringaris, W. Chen, E. Taylor Institute of Psychiatry, London, UK doi: 10.I192/bjp.191.2.182 